\title{
SOLPS5 modelling of the Type III ELMing H-mode on TCV
}

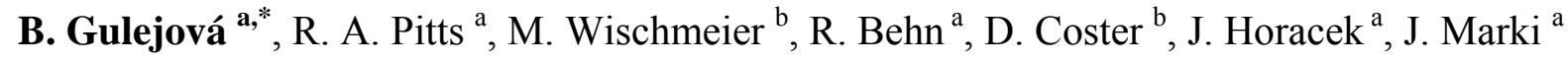 \\ ${ }^{a}$ CRPP-EPFL, Association EURATOM-Confederation Suisse, CH-1015 Lausanne. Switzerland \\ ${ }^{b}$ Max-Planck Institut für Plasmaphysik, EURATOM-Association, Boltzmann Str.2, D-85748, \\ Garching, Germany
}

\begin{abstract}
Although ohmic H-modes have long been produced on TCV and the effects of ELMs at the divertor target studied in some detail, no attempt has yet been made to model the scrapeoff layer (SOL) in these plasmas. This paper describes details of the first such efforts in which simulations of the inter-ELM phases using the coupled fluid-Monte Carlo SOLPS5 code (without drifts) are constrained by careful upstream Thomson scattering and Langmuir probe profiles. Simulated divertor profiles are compared with Langmuir probes and fast IR camera measurements at the targets. To account for the very differing transport rates in the edge pedestal and main SOL regions, radial variation of edge transport coefficients has been introduced in the simulations. Similarly, it is found that transport in the main chamber and divertor regions must be separately adjusted to provide an acceptable code-experiment match.
\end{abstract}

PACS: 52.25.Fi, 52.25.Xz, 52.55.Fa, 52.65.-y

JNM keywords: PO600, TO100

PSI-16 keywords: B2/EIRENE, TCV, Edge modelling, SOL transport, Cross-field transport, Transport barrier, ELM

${ }^{\dagger}$ Present address: Max-Planck Institut für Plasmaphysik, EURATOM-Association, Boltzmann Str.2, D-85748, Garching, Germany

* Corresponding author address: CRPP-EPFL, Association EURATOM-Confederation Suisse, CH-1015 Lausanne. Switzerland

*Corresponding author E-mail: barbora.gulejova@epfl.ch

Presenting author : Barbora Gulejová

Presenting author E-mail : barbora.gulejova@epfl.ch 


\section{Introduction}

Numerical modelling of the tokamak scrape-off layer is essential tool for the prediction of conditions to be expected in future fusion reactors. In particular, transient power loads on plasma-facing components due to energetic plasma relaxations, known as edge localised modes (ELMs), are a major cause of concern in ITER since ELM-target interactions may significantly reduce the expected lifetime of the ITER divertor and even the main chamber walls [1, 2]. However, understanding of the details of ELM-SOL transport and target interactions is still only emerging and considerable effort is required with regard to benchmarking the numerical modelling tools against experimental measurements.

The first step in this exercise is to establish a "steady state" pre- or inter-ELM model to provide a starting point for the more complex time dependent ELM simulations. This paper describes the results of the first effort to provide this for a TCV type-III ELMing H-mode target plasma using the SOLPS5 fluid-Monte Carlo edge code benchmarked against experimental data obtained upstream in the main SOL and pedestal region and at the divertor targets.

Recent experiments with high power ECRH at the third harmonic have produced large, possibly Type I ELMs on TCV for the first time, typically exhausting $\sim 12 \%$ of the plasma stored energy [3], but in standard ohmic H-modes operating close to the L-H transition threshold power, only Type III ELMs are obtained. Typical single null lower (SNL) ohmic Hmode discharges have $\mathrm{I}_{\mathrm{p}}=400 \mathrm{kA}, \mathrm{n} / \mathrm{n}_{\mathrm{GW}} \sim 0.3$ ( $\mathrm{n}_{\mathrm{GW}}$ is the Greenwald density) and steady ELMing phases with $\mathrm{f}_{\text {elm }} \sim 200 \mathrm{~Hz}$, where each ELM exhausts only a few $100 \mathrm{~J}$ of plasma stored energy $(\sim 1-2 \%)$. As such these ELMs cannot be compared with larger events seen on bigger machines regarding the magnitude of target power fluxes etc., but their behaviour with respect to transport in the SOL and interactions with the targets appears to be similar. In fact, their benign nature makes the fluid plasma simulation in some ways more appropriate since the ELMs are insufficiently large to require a true kinetic simulation and are likely to be less perturbing in the sense of parallel heat flux limits and variations in sheath transmission coefficients.

\section{Experiment}

The TCV tokamak $\left(\mathrm{R}=0.875 \mathrm{~m}, \mathrm{a}=0.25 \mathrm{~m}, \mathrm{~B}_{\varphi}=1.43 \mathrm{~T}\right)$, is an all-graphite machine in which a number of open diverted configurations can be achieved. To date, the majority of 
ohmic $\mathrm{H}$-modes have been with reversed toroidal field, $\mathrm{B}_{\varphi}$, (ion $\mathrm{B} \times \nabla \mathrm{B}$ drift direction upwards). This is historical and essentially a consequence of $\mathrm{H}$-mode avoidance, since with the more conventional forward $\mathrm{B}_{\varphi}$ operation, ohmic $\mathrm{H}$-mode is easily obtained, even at low plasma current. This usually occurs without frequent ELMs, making density control difficult. This is particularly important for the low density second harmonic ECRH experiments which constitute a large fraction of the TCV experimental programme.

Simulations described here have been performed for a deuterium Type III ELMing Hmode target discharge (\#26730) with $\mathrm{I}_{\mathrm{p}}=430 \mathrm{kA}, \mathrm{f}_{\mathrm{elm}}=230 \mathrm{~Hz}$ and line averaged electron density, $\bar{n}_{e}=6 \times 10^{19} \mathrm{~m}^{-3}$. The magnetic equilibrium reconstruction of this SNL configuration is shown in Fig. 1, illustrating the short high field side (HFS) divertor leg on the central column and low field side (LFS) strike point on the vessel floor. This rather unconventional diverted configuration is characteristic of TCV and is required principally for reasons of vertical stability. The plasma has elongation and triangularity of $\kappa_{95}=1.65, \delta_{95}=0.4$ respectively, with $\mathrm{q}_{95}=2.5$ and stored energy of $\sim 20 \mathrm{~kJ}$.

The time evolution of a few relevant plasma signals are also shown in Fig. 1 along with the location of the edge diagnostics from which data is used here to constrain the code. Two single Langmuir probe (LP) arrays with time resolution $2.5 \mathrm{~ms}$ and sampling rate $100 \mathrm{kHz}$ provide good coverage of electron temperature $\mathrm{T}_{\mathrm{e}}$, electron density, $\mathrm{n}_{\mathrm{e}}$ and ion flux density, $\mathrm{j}_{\text {sat }}$ at inner and outer targets [4]. During this particular discharge the probes were operated in fixed negative bias mode, generating $j_{\text {sat }}$ on a fast timescale. In further, identical discharges, pre-programmed outer divertor strike points sweeps in conjunction with both voltage sweep and constant bias modes, allow profiles on ion flux, $\mathrm{n}_{\mathrm{e}}$ and $\mathrm{T}_{\mathrm{e}}$ with higher spatial resolution to be generated. Outer target heat flux profiles are deduced from the tile surface temperature using vertically viewing infrared (IR) thermography with time resolution $20 \mathrm{~ms}$ [5]. A fast reciprocating probe $(\mathrm{RCP})$ provides single upstream profiles in the SOL of $T_{e}$ and $n_{e}$ twice in each discharge with a time resolution of $\sim 0.2 \mathrm{~ms}$. An edge Thomson scattering (TS) system provides $T_{e}$ and $n_{e}$ profiles in the pedestal region, including partial coverage of the near SOL and hence some degree of overlap with the RCP data.

\section{Numerical tool (Simulation)}

The SOLPS5 package is a sophisticated coupled fluid (B2.5) and Monte Carlo (EIRENE) code modelling the plasma fluid and neutral transport on a simulation grid generated from the 
measured equilibrium poloidal magnetic flux surfaces from any given tokamak discharge [6]. Experimentally obtained inputs given to the code are magnetic equilibrium, upstream density at the separatrix $\left(1.6 \times 10^{19} \mathrm{~m}^{-3}\right)$ from edge TS and power entering the SOL $\left(\mathrm{P}_{\mathrm{SOL}}=\mathrm{P}_{\Omega}-\mathrm{P}_{\mathrm{RAD}}\right.$ $=330 \mathrm{~kW}$ in this case - see Fig. 1). Since not all data have been available for the discharge we have simulated (\#26730), we have been forced to use the data from several similar discharges. We thus combine the radiated power from \#20703 with edge and SOL profiles from \#26730 (TS and RCP), \#31832 and \#31837 (RCP), \#31838 (LPs) and \#31835 (IR). The value of $\mathrm{P}_{\mathrm{SOL}}$ $=330 \mathrm{~kW}$ estimated from \#20703 is the difference between $\mathrm{P}_{\Omega}=500 \mathrm{~kW}$ and $\mathrm{P}_{\mathrm{RAD}}=225 \mathrm{~kW}$ (see Fig.1). Although the plasma parameters of the two shots \#26730 and \#20703 are very close they were performed at different times with respect to separate vessel boronisations. Since on TCV proximity to boronisation is usually associated with lower impurity levels and hence lower radiation, we expect that $\mathrm{P}_{\mathrm{RAD}}$ for the simulated shot (performed sooner after boronisation than \#20703) would be at least as low as and likely even lower than that assumed (based on \#20703). Since $\mathrm{P}_{\Omega}=555 \mathrm{~kW}$ in the simulated discharge, we expect $\mathrm{P}_{\mathrm{SOL}}=555-$ $225=330 \mathrm{~kW}$ at least. Coefficients, $\mathrm{D}_{\perp}, \chi_{\perp \mathrm{i}, \mathrm{e}}$ controlling the rate of perpendicular particle and energy diffusion are systematically adjusted in order to obtain satisfactory agreement between experimental and simulated midplane profiles (always shown here mapped to the outboard midplane). The transport can also be specified by an adhoc combination of diffusivity and convection, or by convection alone using the convective velocity $\mathrm{v}_{\perp}$, but has not been attempted in this first approach - only $\mathrm{D}_{\perp}, \chi_{\perp \mathrm{i}, \mathrm{e}}$ are varied. In the absence of experimental data for the ion temperature profile (though edge charge-exchange measurements will be available in the future), $\chi_{\perp \mathrm{i}}=\chi_{\perp \mathrm{e}}$ is assumed.

The SOLPS grid extends radially from $-2.5 \mathrm{~cm}$ to $+2 \mathrm{~cm}$ on either side of the midplane separatrix and comprises 72 poloidal and 24 radial cells. This grid extent is sufficient to encompass the edge transport barrier (ETB) region, which is rather narrow in these TCV shots (see below). Albedos are set at 1 on wall surfaces. Carbon is dominant impurity in TCV and is included in the simulation, with transport coefficients assigned as for the fuel species. Parallel heat flux limits are set at 10 for ions (ie essentially no ion heat flux limit), 0.3 for electrons and no drifts have been included at this stage.

The modelling attempts described here broadly follow the approach in the JET EDGE2DNimbus study in [7], seeking first the closest match to upstream experimental profiles during inter-ELM phases using a step-like Ansatz for $\mathrm{D}_{\perp}, \chi_{\perp \text { i,e }}$ in the edge and SOL regions, but also introducing poloidal variation of the transport coefficients such that the transport is specified 
differently in the main chamber SOL and divertor regions. This is extremely important in $\mathrm{TCV}$, where the unconventional divertor geometry means that care must be taken in presence of steep H-mode edge barriers to tailor differently the transport in this region compared with core. Similar reasoning applies even more to the ELM itself, which is known to burst into the SOL in the outboard, unfavourable curvature region and is thus extremely poloidally localised. This poloidal localisation should be accounted for also in the simulations of the ELM itself which may be modelled as an instantaneous local increase in the transport coefficients (as in the earlier SOLPS5 attempts to simulate ELMs in JET [3]) thus simulating the subsequent SOL transport in a time dependent way. This will be the subject of the next stage in the modelling effort described here (in addition to the inclusion of drift flows).

\section{Results and discussion}

\subsection{Upstream profiles}

Fig. 2 compiles the results of the inter-ELM simulation for the upstream profiles of $T_{e}$ and $\mathrm{n}_{\mathrm{e}}$ in comparison to edge TS and RCP data. The TS profiles are compilations of a number of laser pulses through the discharge, filtered to exclude ELM events. The composite profile has been fitted using a tanh function which identifies the experimentally measured pedestal height and width [8] as $3.6 \times 10^{19} \mathrm{~m}^{-3}, 1.36 \mathrm{~cm}$ and $179 \mathrm{eV}, 1.02 \mathrm{~cm}$ for $\mathrm{n}_{\mathrm{e}}$ and $\mathrm{T}_{\mathrm{e}}$ respectively. There is excellent agreement, where the data overlap, between the RCP and TS $\mathrm{T}_{\mathrm{e}}$ profiles. For density, the RCP data have been multiplied by a factor of 0.5 to account for the known overestimation due to finite Larmor radius effects which increase the projected collection area of the Langmuir probe pins. No radial shift has been applied to the profiles.

The SOLPS5 model profiles are also shown in Fig. 2, superimposed on the experimental data. This has been obtained using a radial variation of the transport coefficients as also shown in Fig. 2 and represents an excellent level of agreement for $n_{e}$. In the case of $T_{e}$ it has not been possible to match the full shape of the pedestal even if the separatrix values are close. $\mathrm{D}_{\perp}$ does not require much variation in the region through the confined and ETB regions. However, a gradual decrease of $\chi_{\perp \mathrm{i}, \mathrm{e}}$ is required from just inside the inner boundary of the simulation region right through the ETB. In the main SOL, both $\mathrm{D}_{\perp}$ and $\chi_{\perp \mathrm{i}, \mathrm{e}}$ are increased up to a values of $1 \mathrm{~m}^{2} \mathrm{~s}^{-1}$ and $6 \mathrm{~m}^{2} \mathrm{~s}^{-1}$ respectively. A higher value of $\chi_{\perp \mathrm{i}, \mathrm{e}}=6 \mathrm{~m}^{2} \mathrm{~s}^{-1}$ in the main SOL has been applied in order to flatten the temperature profile there. Interestingly, 
this is consistent with the findings of a similar simulation exercise recently reported from ASDEX-Upgrade [9]. It seems clear that the foot of the ETB extends someway $(\sim 0.5 \mathrm{~cm}$ at the midplane) into the SOL itself. This is similar to the JET findings in [7]. Unlike the analysis in [7], however, no inward pinch nor outward drift has been found necessary to model the experimental profiles, though it is clear that there is a certain arbitrariness to the choice of these coefficients, since closely similar results can be found by introducing a profile of $\mathrm{v}_{\perp}$ and appropriately adjusting those of $\mathrm{D}_{\perp}$ and $\chi_{\perp \mathrm{i}, \mathrm{e}}$. Furthermore, the derived SOLPS5 transport parameters $\mathrm{D}, \chi$ have been compared with ion neoclassical values in the pedestal region (calculated using the density and temperature data from SOLPS since pedestal measurements of ion temperature are not yet available on TCV) and found to be very similar.

\subsection{Target profiles}

Fig. 3 presents the measured inner and outer divertor target $j_{s a t}, n_{e}$ and $T_{e}$ profiles compared with the simulation for the upstream match shown in Fig. 2. At the outer target the experimental parallel ion fluxes have been obtained both from fast voltage sweep of the probes as the divertor leg itself is swept through the steady ELMing phase (see Fig. 1) and by extracting the pre-ELM $\mathrm{j}_{\text {sat }}$ value obtained from a coherent ELM average (following the technique reported in [4]) on a probe by probe basis for a case with the outer strike point fixed in time (in a separate, identical discharge). The two techniques provide similar profiles, though the divertor leg sweep clearly has the advantage of offering higher spatial resolution. Data at the inner target are of lower quality - floating potentials of the inner target LP, especially in the strike point region, are very negative (due to the presence of strong thermoelectric currents [4]) and insufficient bias potential often makes it difficult to extract $\mathrm{T}_{\mathrm{e}}$ reliably.

In general, the simulation experiment match is again satisfactory, but it should be noted that this has only been possible by "switching off" the transport barrier in the divertor regions and increasing setting values of $\mathrm{D}_{\perp}$ and $\chi_{\perp \mathrm{i}, \mathrm{e}}$ in the divertor SOL and private flux region (PFR) from $1 \rightarrow 6 \mathrm{~m}^{2} \mathrm{~s}-1$ to $3 \mathrm{~m}^{2} \mathrm{~s}^{-1}$ and $5 \mathrm{~m}^{2} \mathrm{~s}^{-1}$ respectively $\left(\mathrm{cf} . \mathrm{D}=1 \mathrm{~m}^{2} \mathrm{~s}^{-1}\right.$ and $\chi_{\perp \mathrm{i}, \mathrm{e}}=6 \mathrm{~m}^{2} \mathrm{~s}^{-1}$ in the main chamber SOL - Fig. 2). As can be seen from the model profile widths at the outer target, the PFR transport should probably be decreased to steepen the profile there.

Without the increased divertor transport rates, the code overestimates the experimental outer target $j_{\text {sat }}$ and $T_{e}$ by more than a factor of 3 , but it should be clear that because drifts 
have not been included in the simulation, it is somewhat artifical to seek too close a match. Drifts very likely are playing a role in $\mathrm{TCV}$, but the situation is complicated by the unconventional divertor geometry combining a short and long divetor leg. In reversed $\mathrm{B}_{\varphi}$ (the case considered here), observations made elsewhere (eg on the JET [10] and Alcator C-Mod [11] tend to find that although $\mathrm{T}_{\mathrm{e}}$ at the inner usually stays lower than at the outer, the difference is significantly reduced compared with forward $B_{\varphi}$, where $T_{e}$ is always much higher at the outer target. This is also of course due to toroidal geometry which automatically ensures that more power flows to the outer SOL.

In TCV, the experimental data in Fig. 3 indicate a hotter inner divertor in reversed $\mathrm{B}_{\varphi}$, but some of this is undoutbtedly due to the short inner divertor leg. The code approximately matches the inner target $T_{e}$ but overestimates that at the outer by about a factor of 2 . The code slightly underestimates the inner target $\mathrm{T}_{\mathrm{e}}$ but matches well that of outer. Density, which is reasonably well matched at both targets, is computed from the probe characteristic fits as $n_{e}=$ $\mathrm{j}_{\text {sat }} / \mathrm{e} \mathrm{c}_{\mathrm{s}}$, with the $\mathrm{c}_{\mathrm{s}}$ the sound speed assuming $\mathrm{T}_{\mathrm{i}}=\mathrm{T}_{\mathrm{e}}$. The code simulations indicate that the latter is a good approximation at the target. Switching on drifts (the subject of planned future work) is very likely to decrease the predicted outer target $T_{e}$ and may have only a small effect at the inner target (for the geometrical reasons mentioned above). In fact, very recent experimental observations of forward $\mathrm{B}_{\varphi}$ ohmic H-modes, albeit at lower current $\left(\mathrm{I}_{\mathrm{p}}=330\right.$ kA) than the discharge considered here have shown that the experimental outer target $T_{e}$ is increased by almost a factor 2 over the values shown here, which would match these drift-free SOLPS5 simulations.

Fig. 4 compares the experimental LP and predicted perpendicular target power fluxes, $\mathrm{P}_{\perp}$ for both targets where, at the outer target, new data from a vessel floor viewing IR camera have been included [5]. The LP and SOLPS5 estimates are computed according to $\mathrm{P}_{\perp}=$ $\gamma \mathrm{j}_{\mathrm{sat}} \mathrm{T}_{\mathrm{e}} \sin \alpha$ with $\alpha$ the angle of incidence of the total magnetic field at the targets and $\gamma$ the sheath heat transmission coefficient. The latter has been assumed as 7.5 for the purposes of Fig. 4, but comparison with the IR profile shows that slightly higher values would be more appropriate in this case [5]. Due principally to the overestimate of target $T_{e}$, SOLPS5 overestimates the outer target power ( $\sim$ factor 2$)$, but is only a factor 1.12 above the measured IR profile and slightly underestimates the inner target values. The integral experimental power balance (summing the inner and outer target contributions to obtain $\mathrm{P}_{\mathrm{SOL}}$ ) is quite well satisfied but slightly overestimates the expected power. Fig. 4 clearly shows that the majority of the conducted power is found at the inner target in this configuration. This is confirmed by 
tomographic inversion of chordal radiation measurements which show the strongest radiating zone to be located at the $\mathrm{X}$-point and inner strike regions.

\section{Conclusions}

First attempts have been made to model the inter-ELM SOL plasma of ohmic ELMing Hmode on TCV using the SOLPS5 coupled fluid-Monte Carlo code package. Simulations are constrained by seeking to match upstream SOL and edge pedestal profile measurements. No such match is possible without introducing radial variations of the particle and heat diffusivity, with low values in the edge transport barrier region and an increase in the SOL. Compared with the main chamber SOL values, $\mathrm{D}_{\perp}$ must be increased roughly fivefold threefold and $\chi_{\perp \mathrm{i}, \mathrm{e}}$ remains approximately the same if outer target experimental profiles of ion flux and $T_{e}$ are to be even approximately matched. Agreement between simulation and experiment is generally good at both targets, though the code predicts lower $T_{e}$ at the inner target, where densities and temperatures are considerably higher than at the outer. At least part of the reason for the higher code values, particularly of $\mathrm{T}_{\mathrm{e}}$, found at the outer target in comparison with experiment is due to the neglect of drift effects in these first simulations. The reasonably good match at the inner target may be an indication that it is the rather unconventional equilibrium, with an extremely short X-point to inner strike point position, which dominates over any drift or divertor physics effects. The encouraging overall modelexperiment agreement for this steady state phase provides a good basis for the more complex time dependent modelling of the ELM itself.

\section{Acknowledgements}

This work was partly supported by the Swiss National Science Foundation.

\section{References}

[1] R. A. Pitts et al., PPCF 47 (2005) B303

[2] G. Federici et al., PPCF 45 (2003) 1532

[3] Y. Martin et al., PPCF 48 (2006) A163 
[4] R. A. Pitts et al., Nucl. Fusion 43 (2003) 1145

[5] J. Marki et al., PSI 17, Hefei, 2006

[6] R. Schneider et al., Contribution to Plasma Physics 46 (1) (2006) 3-191

[7] A. Kallenbach et al., Plasma Phys. Control Fusion 46 (2004) 431

[8] A. Alfier et al., 32 ${ }^{\text {nd }}$ EPS, Tarragona, ECA 29C, P-2.002 (2005)

[9] A. V. Chankin et al., Plasma Phys. Control Fusion 48 (2006) 839 - 868

[10] R. A. Pitts et al., J. Nucl. Mater. 337-339 (2005) 146

[11] I. H. Hutchinson et al., PPCF 37 (1995) 1389 


\section{Figure captions}

Fig.1 Magnetic equilibrium reconstruction of \#26730 (for which the SOLPS5 grid is generated) showing the locations of edge diagnostics from which data are compared with the simulations. Relevant plasma signals for this discharge are shown at right: $D_{\alpha}$ emission (measured along a vertical LOS centred on the magnetic axis), average ELM frequency $\sim 230$ Hz. $\mathrm{P}_{\mathrm{SOL}} \sim 330 \mathrm{~kW}$ is estimated using radial power from the identical discharge \#20703 and the assumption that $\mathrm{P}_{\mathrm{RAD}}$ in \#26730 is at least as low as that in \#20703.

Fig.2. $n_{e}$ and $T_{e}$ upstream profiles from SOLPS5, edge TS and RCP. The perpendicular particle and heat transport coefficients used to obtain the upstream match are shown in the lower plot. All data have been mapped to the outer midplane and expressed in terms of distance from the separatrix $\left(r-r_{\text {sep }}=0\right)$. The RCP data are from shots \#26730, \#31832 and \#31837 and the TS data from \#26730. SOLPS simulation number is 16418.

Fig.3. $\mathrm{j}_{\mathrm{sat}}, \mathrm{T}_{\mathrm{e}}$ and $\mathrm{n}_{\mathrm{e}}$ profiles at inner (left) and outer target (right) from SOLPS5 (simulation 16418) and LP. Probe data at the outer target are obtained during an outer divertor leg sweep (\#31838) with the exception of the $j_{\text {sat }}$ points marked with squares which result from a coherent ELM average in a separate discharge without strike point sweeping. They act as a cross-check of the swept data.

Fig.4. Perpendicular heat flux at the inner (top) and outer target (bottom) from SOLPS5 (simulation 16418), LP and IR camera (outer target only), where data are taken from \#31838 for LP and \#31835 for IR. 
P2-7

Figures

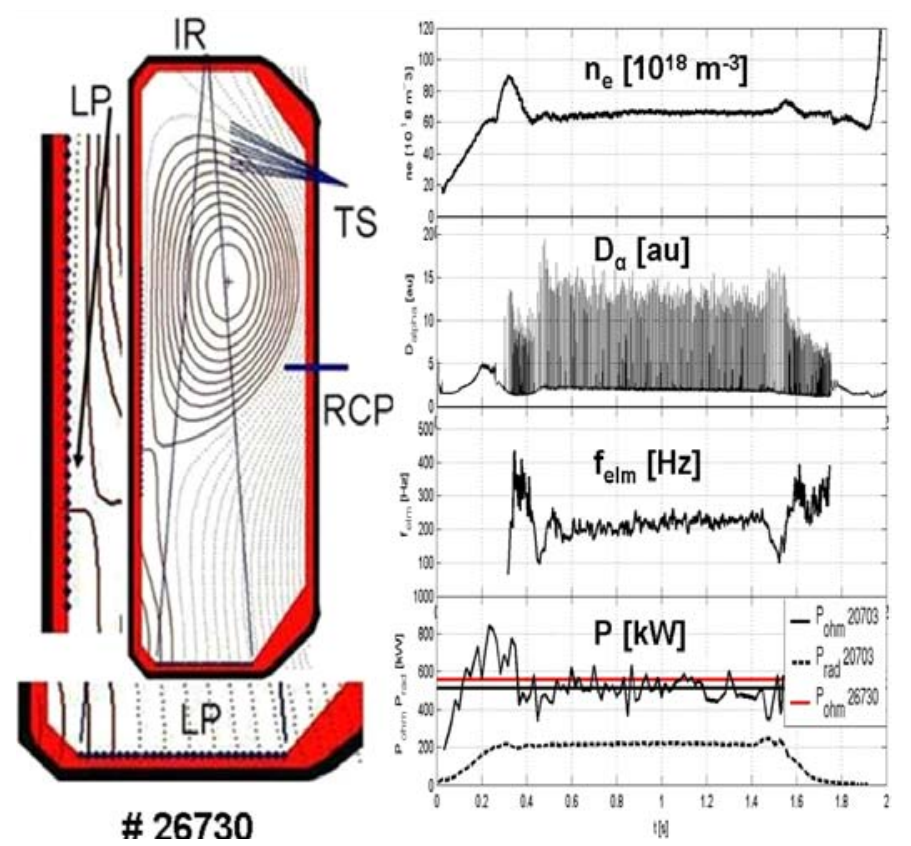

Figure 1 


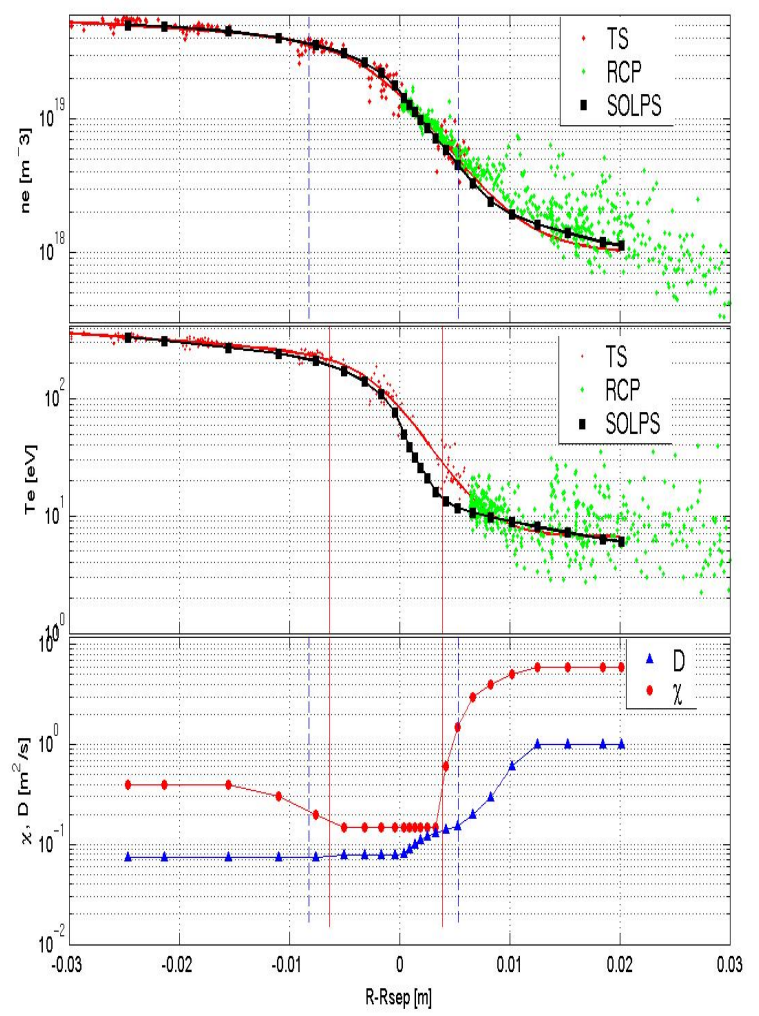

Figure 2

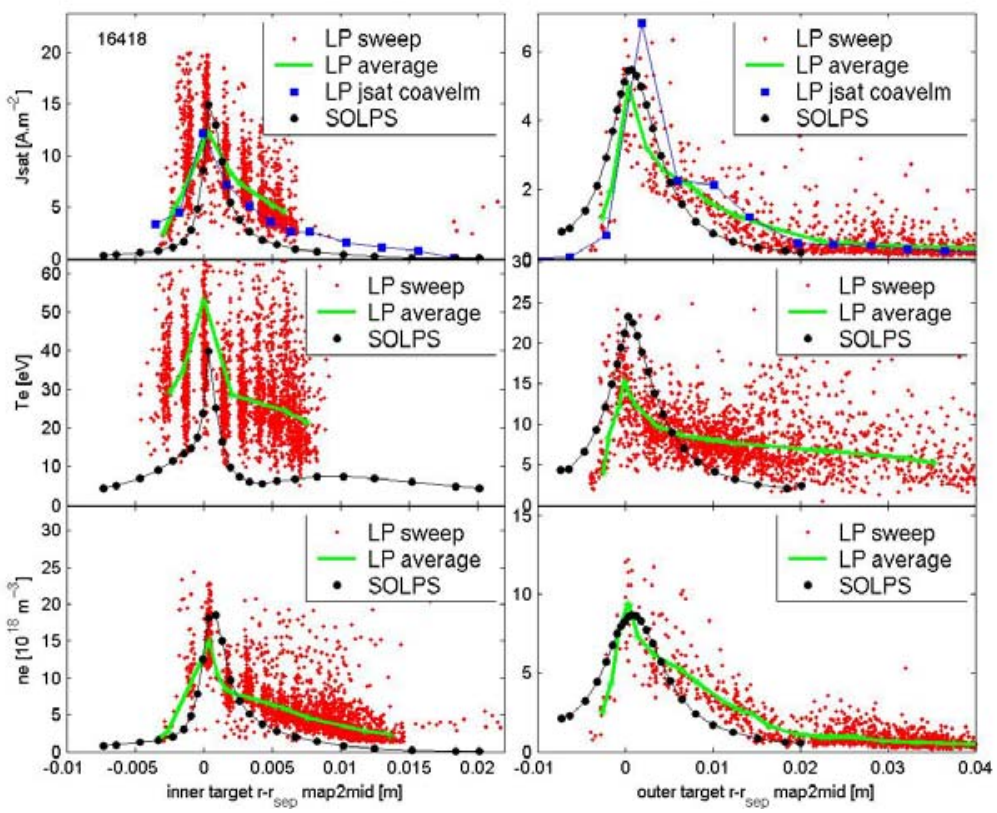

Figure 3 


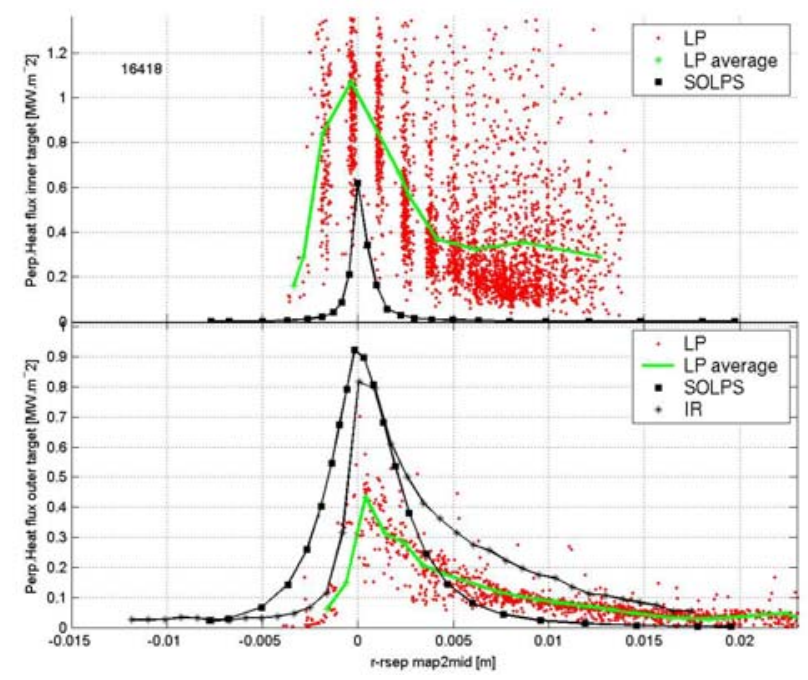

Figure 4 